\title{
Human and livestock trematode infections in a mobile pastoralist setting at Lake Chad: added value of a One Health approach beyond zoonotic diseases research
}

\author{
Helena Greter ${ }^{a, b, *}$, Annour A. Batil ${ }^{c}$, Bongo N. Ngandoloc, Idriss O. Alfaroukhc, Doumagoum D. Moto ${ }^{d}$, \\ Jan Hattendorf ${ }^{a, b}$, Jürg Utzinger ${ }^{a, b}$ and Jakob Zinsstag ${ }^{a, b}$
}

${ }^{a}$ Swiss Tropical and Public Health Institute, PO Box, CH-4002 Basel, Switzerland; ' University of Basel, Petersplatz 1, CH-4003 Basel, Switzerland; ' Institut de Recherche en Elevage pour le Développement, N'Djamena, Chad; 'Centre de Support en Santé International, N'Djamena, Chad

*Corresponding author: Tel: +4161 284-8730; E-mail: helena.greter@swisstph.ch

Received 17 March 2017; revised 10 May 2017; editorial decision 3 August 2017; accepted 4 August 2017

\begin{abstract}
Background: At Lake Chad in Central Africa, mobile pastoralists face economic losses due to livestock trematodiases. Fasciola gigantica and Schistosoma bovis-trematodes that affect livestock-share transmission ecology traits with Schistosoma haematobium and S. mansoni that cause human schistosomiasis. This project aimed at assessing treatment strategies and elucidating the predictive potential of human and livestock trematode infections.
\end{abstract}

\begin{abstract}
Methods: Schistosomiasis and fascioliasis were investigated concurrently in humans and cattle by repeated cross-sectional surveys. Urine and stool samples from humans and faecal samples from cattle were examined for trematode eggs. Treatment strategies were assessed by means of focus group discussions and in-depth interviews.

Results: Mobile pastoralists of four ethnic groups participated. Prevalence of human schistosomiasis and livestock trematodiases showed considerable heterogeneity from one ethnic group to another, but correlated within ethnic groups. Effective trematocidal drugs were not available in the study area.

Conclusions: Mutual predictive potential of human schistosomiasis and livestock fascioliasis relates to distinct livestock husbandry practices. Introducing efficacious strategic treatment against human schistosomiasis and livestock fascioliasis might improve human and animal health and well-being. Our research provides evidence for the benefits of a One Health approach targeting diseases that share specific ecological traits.
\end{abstract}

Keywords: Chad, Disease ecology, Fascioliasis, Mobile pastoralists, One Health, Schistosomiasis

\section{Introduction}

In the frame of a long-standing research partnership built on mutual trust, a multi-stakeholder workshop was held in N'Djamena, Chad in 2011. It brought together pastoralists, representatives of the ministries of health and livestock production, scientists, and other constituencies in a trans-disciplinary process. ${ }^{1}$ Pastoralists emphasized the importance of fascioliasis as a veterinary health problem affecting livestock productivity, thereby causing economic losses and having a negative impact on their livelihood. ${ }^{2}$

At Lake Chad, livestock fascioliasis is caused by Fasciola gigantica, a water-related liver fluke depending on a freshwater snail as intermediate host in its life cycle, similar to the blood flukes of the genus Schistosoma. Livestock schistosomiasis is caused by Schistosoma bovis, while S. haematobium and S. mansoni are the causative agents for human schistosomiasis. Infection with $F$. gigantica occurs when ruminants are grazing on contaminated pasture in close proximity to open freshwater bodies, whereas Schistosoma spp. infect humans and animals by actively penetrating the skin while individuals are in direct contact with contaminated water. Given the shared transmission ecology and similarities in the life cycle of the four trematode species, we concurrently investigated the epidemiology of fascioliasis and schistosomiasis in humans and livestock in a mobile pastoralist setting at Lake Chad. To generate a more holistic picture of the prevailing disease situation in that specific setting, treatment strategies and access to and availability of trematocidal drugs were also assessed. 
Throughout the Sahelian belt, mobile pastoralism is the predominant livestock husbandry system providing livelihoods for an estimated 25 million people and producing milk and meat for many more. ${ }^{3}$ In an arid environment with a limited suitability for agricultural activities, raising livestock by following the annual cycle of pasture growth-known as transhumance-represents a highly adapted and sustainable lifestyle. ${ }^{4}$ The flexibility of the mobility results in a high level of resilience and provides the means to cope with uncertainty and subtle changes in the environment, where rainfall and the consecutive growth of vegetation determine human and livestock movements. The main limiting factor is water and water availability. In that specific ecosystem, human and livestock populations share benefits and risks of water contacts. Indeed, surface water is used for human consumption, as well as for watering of livestock, for washing and bathing, and humans and livestock are crossing open water during migration. ${ }^{5,6}$

When looking at the human and animal exposure patterns from an integrated One Health perspective, it is hypothesized that the risk of infection with water-related parasites in humans and animals is comparable. Considering the biological and ecological similarities in the life cycles of F. gigantica on one hand, and S. bovis, S. haematobium, and S. mansoni on the other hand, this project aimed at testing whether there is a mutual predictive potential of F. gigantica infection in livestock and Schistosoma infection in humans and vice versa. Livestock fascioliasis can be detected in live animals through parasitological methods by performing the sedimentation technique and microscopy on faecal samples, although this is rarely practised and not applied at all in the Lake Chad area. More commonly, the disease is monitored at slaughter; adult F. gigantica flukes can reach a body length of up to
$7 \mathrm{~cm}$ and are thus easily detectable in contaminated animal livers at meat inspection. In contrast, the diagnosis of human schistosomiasis depends on laboratory diagnosis, which is not available in remote rural settings of the Lake Chad area. ${ }^{7}$ If a predictive potential from livestock fascioliasis to human schistosomiasis can be identified, this would present scientific evidence for the use of animal health data (e.g. fascioliasis in cattle) as sentinel for human schistosomiasis in a setting where no schistosomiasis control programme is in place, as is the current situation at Lake Chad. ${ }^{8}$

This article summarizes findings from a One Health study on trematode infections in a mobile pastoralist setting at Lake Chad, highlighting the potential of a One Health study design beyond direct application to zoonotic diseases, to pathogens sharing similarities in their transmission ecology. It reports on the feasibility and efficiency of a joint human and animal research study design for water-based parasitic infection epidemiology. We discuss future perspectives and integrative potential with further innovative approaches for joint human and animal health research for pathogens with similar transmission ecology.

\section{Methods}

\section{Study area}

This study was carried out within the semi-arid Sahelian zone on the eastern shores of Lake Chad on Chadian territory (Figure 1). Lake Chad shows seasonally oscillating water levels and is surrounded by a large flooding zone. The seasonal inundations play a crucial role in the evolution of migratory routes of mobile pastoralists. Towards the end of the dry and hot season, pasture only remains on Lake Chad's islands and shores, and mobile

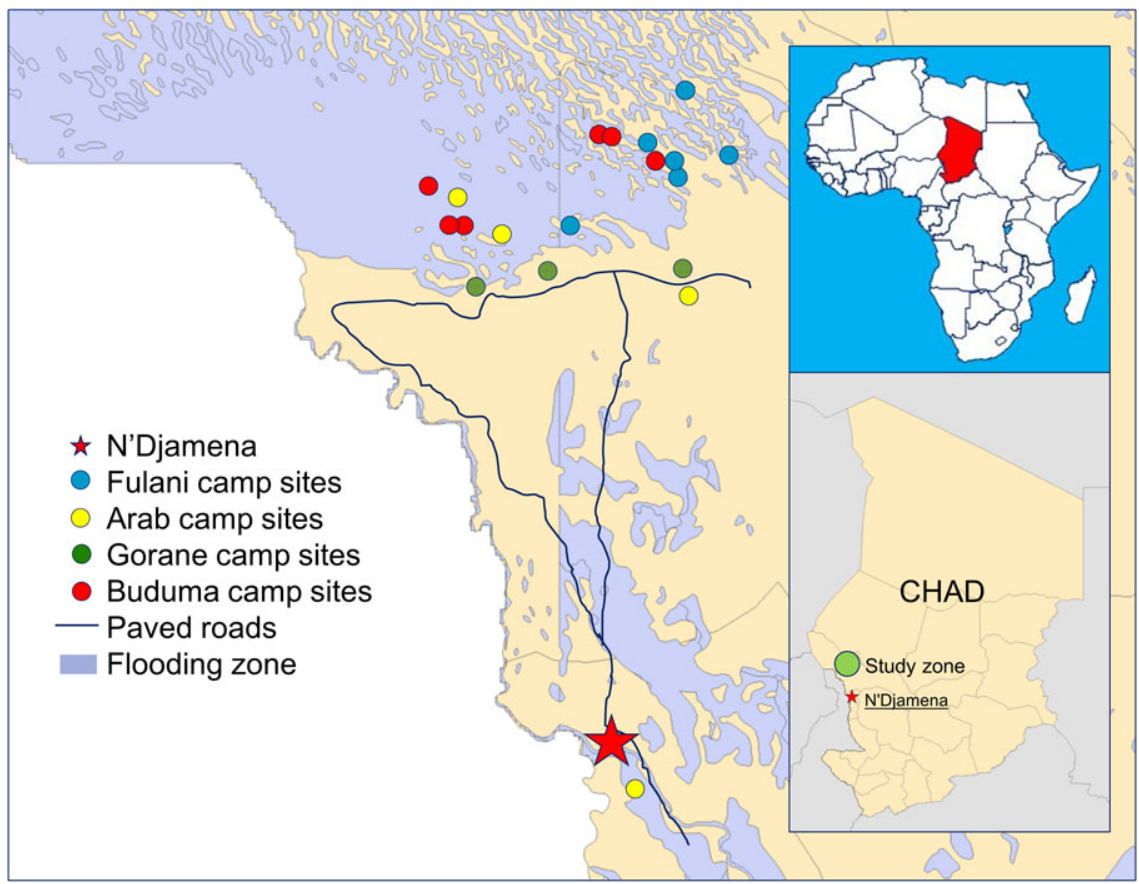

Figure 1. Map of the study zone on the eastern shores of Lake Chad, indicating the camp sites of the 19 participating groups of mobile pastoralists. Also displayed is the maximum surface area of Lake Chad, including the flood zones, which can be submerged after the rainy season, and dry up during the dry season. 
pastoralists of different ethnic groups together with their flocks gather in the area. $^{9}$

\section{Study population}

Mobile pastoralists belonging to one of the four predominant ethnic groups in the area were invited to participate. Among the four ethnic groups, the Fulani, Buduma, and Gorane are living a fully mobile lifestyle, while the Arabs are agro-pastoralists. The four ethnic groups differ in cultural aspects such as language and housing styles, but also in transhumance strategy, husbandry system, and cattle breed herded. ${ }^{5}$ Predominant cattle breeds are the Shewa (Arab Zebu), the Mbororo (Red Fulani), and the Kouri cattle. ${ }^{10,11}$

\section{Study design}

The study consisted of repeated cross-sectional surveys. Data collection took place from September 2013 to December 2014. Due to the lack of demographic data on the mobile pastoralist population in the study area, there was no sampling frame allowing for a sampling proportional to size.

The sample size was determined assuming a prevalence of $5 \%$ and an intra-class correlation coefficient of 0.2 . Hence, we required 20 clusters with 20 human participants and 20 cattle in each cluster to estimate the prevalence with a precision of the sample estimate (defined as one half-length of the $95 \%$ confidence interval (CI)) of $5 \%$ points. The number of clusters and the number of samples per cluster depended also on the prevailing cultural practices and the logistical feasibility in a remote location with poor security standards and threat of incursions of terrorist groups.

Individual participants were randomly selected amongst the group by using the previously published spatial sampling method from the Expanded Programme of Immunization (EPI) of the World Health Organization (WHO). In brief, starting from a central point in the camp site, a direction was randomly selected and followed to the nearest household. From this first household, the second nearest household was selected. ${ }^{12}$ Within each randomly selected household, two participants were selected by counting out random numbers. This procedure was repeated until 20 participants were enrolled. All individuals aged above 5 years were eligible for participation. Among the cattle herd of the pastoralist group, 20 animals aged 1 year and above were randomly selected by counting out random numbers and were individually ear tagged.

\section{Parasitological survey}

Urine filtration, reagent strip testing for microhaematuria, and point-of-care circulating cathodic antigen (POC-CCA) urine cassette test, as well as microscopy of Kato-Katz thick smears from stool samples were performed in a mobile field laboratory in proximity to the pastoralists' camp sites. Additionally, stool samples were fixed in sodium acetate-acetic acid-formalin (SAF) for transfer and subsequent ether-concentration preparation at the Swiss Tropical and Public Health Institute (Swiss TPH) in Basel. In cattle, a faecal sample was collected from each animal and fixed in SAF for subsequent analysis with the sedimentation technique in laboratories in N'Djamena, Chad and Zurich, Switzerland.

\section{Assessment of treatment availability and access}

During questionnaire interviews with individuals infected with Schistosoma, disease perception, treatment strategies, healthseeking behaviour, treatment outcome, and satisfaction were assessed. Livestock fascioliasis treatment strategies and outcome satisfaction were addressed during focus group discussions. Locally available trematocidal drugs were tested for their active ingredient and its quantity using a high pressure liquid chromatography (HPLC)-UV method, combined with tandem mass spectrometry, of which the detailed results are reported elsewhere. ${ }^{13}$

\section{Statistical analysis}

Prevalence estimates were adjusted for correlation within clusters using generalized estimating equations for binary outcomes.

\section{Ethical considerations}

The study was approved by the ethics committee in Basel [EKBB; reference no. 64/13]. In Chad, research permission, including ethical approval, was obtained from the 'Direction Générale des Activités Sanitaires' [reference no. 343/MSP/SE/SG/ DGAS/2013]. The aim and procedures of the study were explained to each group of mobile pastoralists. Informed consent was signed by group leaders after discussion within the groups. Because of high illiteracy rates, randomly selected individuals within each group provided oral assent.

All participants with a positive diagnostic test from either urine filtration, POC-CCA urine cassette test, or Kato-Katz thick smear at the mobile field laboratory received treatment with a single oral dose of praziquantel $(40 \mathrm{mg} / \mathrm{kg})$ against schistosomiasis and albendazole $(400 \mathrm{mg})$ against soil-transmitted helminthiasis, administered by the teams' medical staff. All selected cattle received a single oral dose of triclabendazole (12 mg/kg).

\section{Results}

\section{Parasitological survey}

A total of 19 groups of mobile pastoralists belonging to the four ethnic groups of the Fulani, Buduma, Arab, and Gorane participated. Of the 415 participants enrolled, 369 were subjected to full urine analyses (urine filtration, reagent strip testing, and POC-CCA) and 284 for full stool analyses (Kato-Katz thick smear on fresh samples and ether-concentration on SAF-fixed samples). Individuals with complete parasitological data were included in further analyses. Regarding cattle, faecal samples of 534 animals were analysed.

Overall, S. haematobium was the most prevalent trematode infection in humans, with a prevalence of $8.1 \%(95 \%$ CI: 5.0-12.8\%). Stool sample examinations revealed an S. mansoni prevalence of $0.4 \%$ (95\% CI: $0.05-2.4 \%)$ with a single participant from the Buduma ethnic group being tested positive, whereas POC-CCA urine cassette tests for S. mansoni showed a prevalence of $9.2 \%$ (95\% CI: 5.1-16.2\%). In cattle, F. gigantica prevalence was $31.3 \%(95 \%$ CI: $23.3-40.6 \%)$ and S. bovis prevalence was $20.3 \%$ (95\% CI: $13.0-30.2 \%$ ) (Table 1).

Stratification revealed that trematode prevalence varied significantly between ethnic groups, but showed correlation in 
Table 1. Prevalence of human and animal trematode infections, stratified by sex and ethnic group (humans), and by sex and husbandry system (cattle)

\begin{tabular}{|c|c|c|c|c|c|c|c|c|}
\hline \multirow{2}{*}{$\begin{array}{l}\text { Prevalence of human trematode infections } \\
\text { Urine }\end{array}$} & \multirow[b]{2}{*}{ Total } & & \multicolumn{2}{|l|}{ Sex } & \multicolumn{4}{|c|}{ Ethnic group } \\
\hline & & & Male & Female & Gorane & Arab & Buduma & Fulani \\
\hline$N(\%)$ & 369 & & $186(50.5)$ & $183(49.5)$ & $61(16.5)$ & $79(21.4)$ & $105(28.5)$ & $124(33.6)$ \\
\hline Prevalence \% (n positive) & & $95 \%$ CI & & & & & & \\
\hline S. haematobium & 8.1 (29) & $5.0-12.8$ & $8.6(16)$ & $7.1(13)$ & $1.6(1)$ & $8.9(7)$ & $10.4(11)$ & $8.1(10)$ \\
\hline Stool & Total & & Male & Female & Gorane & Arab & Buduma & Fulani \\
\hline N (\%) & 284 & & $144(50.7)$ & $140(49.3)$ & $49(17.2)$ & $83(29.2)$ & $59(20.8)$ & $93(32.8)$ \\
\hline Prevalence $\%$ (n positive) & & $95 \%$ CI & & & & & & \\
\hline S. mansoni & $0.4(1)$ & $0.05-2.4$ & $0.7(1)$ & 0 & 0 & 0 & $1.7(1)$ & 0 \\
\hline \multirow[t]{2}{*}{ Prevalence of bovine trematode infections } & & & \multicolumn{2}{|l|}{ Sex } & \multicolumn{4}{|c|}{ Husbandry system } \\
\hline & Total & & Male & Female & Gorane & Arab & Buduma & Fulani \\
\hline$N(\%)$ & 534 & & $81(15.2)$ & $453(84.8)$ & $84(15.7)$ & $126(23.6)$ & $161(30.2)$ & $163(30.5)$ \\
\hline Prevalence \% (n positive) & & $95 \%$ CI & & & & & & \\
\hline F. gigantica & $31.3(167)$ & $23.3-40.6$ & $28.8(23)$ & $31.9(114)$ & $1.1(1)$ & $24.6(31)$ & $36.0(58)$ & $47.3(77)$ \\
\hline S. bovis & $20.3(114)$ & $13.0-30.2$ & $22.5(18)$ & $21.3(96)$ & $3.4(3)$ & $17.5(22)$ & $29.2(47)$ & $25.8(42)$ \\
\hline
\end{tabular}

humans and cattle within ethnic groups. Whereas in Gorane pastoralists groups there were only single human and cattle schistosomiasis and fascioliasis cases, in Fulani and Buduma pastoralists, both humans and their cattle showed considerable infection rates. Arab pastoralists and their cattle showed intermediate schistosomiasis and fascioliasis prevalence (Figure 2).

\section{Assessment of treatment availability and access}

Among the schistosomiasis cases, $>60 \%$ sought treatment in health centres and from the informal market. Fascioliasis awareness among pastoralists was high and self-mediated therapy using veterinary drugs is the common practice. Mebendazole and albendazole are the drugs locally available, which are used against human parasitic worm infections and for livestock fascioliasis treatment. No praziquantel or triclabendazole-the current treatments of choice against schistosomiasis and fascioliasis, respectively-were among the drug samples obtained on the local markets. ${ }^{13}$

\section{Discussion}

Our research was embedded in an established partnership and initiated through a trans-disciplinary process, which allowed us to identify health priorities in the population studied and to come forward with specific research questions. Indeed, mobile pastoralists, local authorities, scientists and other stakeholders engaged in a dialogue that yielded fascioliasis as one of the most important prevailing animal health problems. Knowing about the similarities in the disease ecology and epidemiology of fascioliasis and schistosomiasis, this project investigated the human and livestock diseases concurrently, with the aim to explore potential starting points to develop effective and targeted joint human and animal health interventions.

We found considerable differences in the prevalence of human and cattle schistosomiasis and livestock fascioliasis between the four ethnic groups, whereas distribution patterns of human and animal trematode infections within ethnic groups were similar (Figure 2). Traditionally evolved mobility patterns, transhumance practices, and type of livestock husbandry systems differ between the ethnic groups, which might govern distinct exposure risks for acquiring trematode infections for humans and cattle alike. Specifically, access to and use of open freshwater bodies varies between the four ethnic groups, which in turn might explain the prevalence of human and animal trematode infections. For example, the Buduma and Fulani pastoralists grazing their animals inside the flooding zone expose themselves and their livestock to a higher risk of infection with Schistosoma spp. and F. gigantica than Arab and Gorane pastoralists who remain largely in the dry lands surrounding the lake (Figure 1). While the Gorane pastoralists avoid grazing and watering animals at open surface water and prefer to use water from traditional wells, the Fulani and Buduma pastoralists use surface water and pastures on the shores and islands of Lake Chad year-round. The Arab agropastoralists live in villages where they use traditional wells and pumps, and only approach Lake Chad's shores when the pasture around their villages is fed down towards the end of the dry season. This is also the period when they use surface water for human consumption and watering of livestock. ${ }^{5}$ 


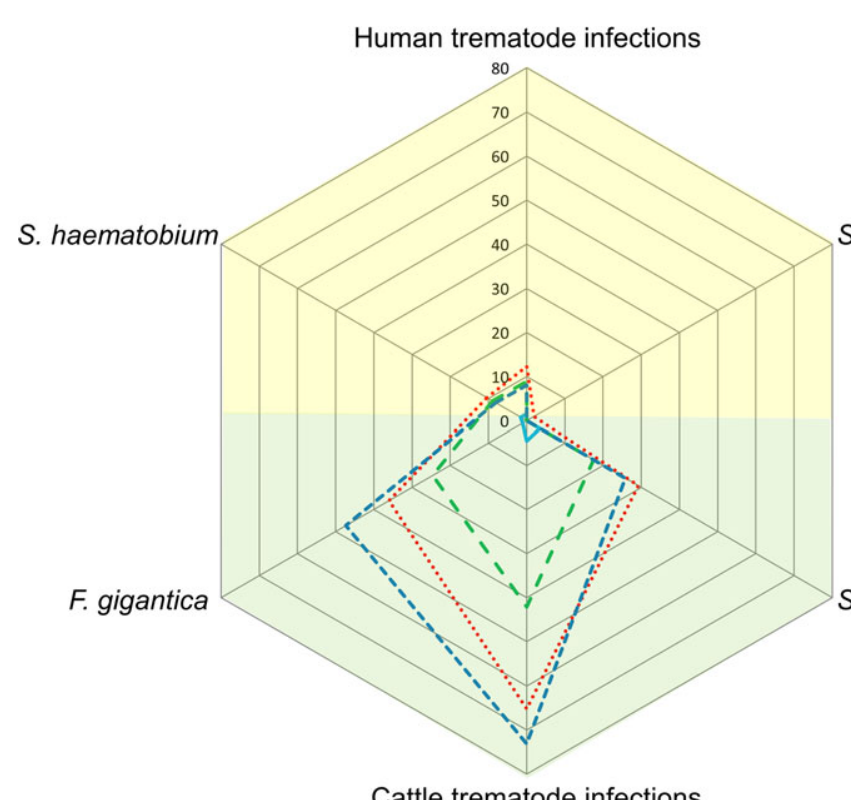

Cattle trematode infections
S. mansoni

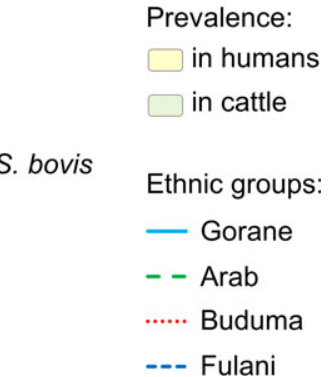

Figure 2. Prevalence of human and livestock schistosomiasis and fascioliasis in a mobile pastoralist setting at Lake Chad, stratified by ethnic group and the respective husbandry system.

Interventions for the control of fascioliasis and schistosomiasis should thus focus on Fulani, Buduma, and Arab pastoralists and their cattle. For both human and animal trematode infections, the most effective drugs-praziquantel and triclabendazolewere not available in the study zone. ${ }^{13}$ Introducing efficacious treatments against human schistosomiasis and livestock fascioliasis might not only positively impact human and animal health, but might also result in economic benefits by improving livestock productivity and reducing treatment costs.

The high fascioliasis prevalence in live animals shown here confirms findings of a previous abattoir study. ${ }^{2}$ Additionally, S. bovis infection in cattle was found at a prevalence above $20 \%$. Besides a report from the 1970s, to our knowledge, the current study provides new evidence that bovine schistosomiasis is endemic in the Lake Chad area. ${ }^{11}$ However, there was limited awareness of the disease among Chadian livestock breeders, veterinarians, and technicians. This might be explained by the fact that clinical manifestations in naturally infected animals are not well documented and are assumed to be subclinical and/or chronic. ${ }^{14}$ It therewith receives no veterinary attention and no treatment is administered. ${ }^{15}$ This leads to the conclusion that pastoralists erroneously attribute the morbidity caused by bovine schistosomiasis to fascioliasis and the subsequently administered anti fascioliasis treatment fails to improve the animals' condition, which lead to low satisfaction with the available treatment. Given the high prevalence reported here, the epidemiology of livestock schistosomiasis seems largely underappreciated in Chad. Indeed, there is a paucity of prevalence data in the extent literature and most publications solely report on occurrence. ${ }^{16-18}$ Surprisingly, more data pertaining to molecular biology are available than on the epidemiology of livestock schistosomiasis. ${ }^{19}$ In recent years, research on $S$. bovis has gained traction, since molecular investigations revealed hybridization with the human infective species S. haematobium. ${ }^{20}$ As a prominent example, the
2013 outbreak of schistosomiasis on the French island of Corsica is worth mentioning. ${ }^{21}$ As Calvatas and Martin ${ }^{14}$ reminded in a letter, bovine schistosomiasis had been reported from Corsica until the 1960s, which shows that the intermediate host snails are present and the parasite's life cycle could be completed in Corsica.

The findings of our One Health study revealed the spatial and temporal co-occurrence of S. haematobium, S. mansoni, and S. bovis in the Lake Chad area. Such co-occurrence could potentially promote hybridization of $S$. haematobium and S. bovis, leading to novel phenotypic properties in the hybrid parasites. Research on existing or on-going hybridization is of considerable interest, specifically in light of current efforts to move from morbidity control towards interruption of schistosomiasis, hence aiming at local elimination. ${ }^{22} \mathrm{~A}$ potential associated host switch could change the known epidemiology of human schistosomiasis. Control and elimination programmes would then need to be expanded to include the animal hosts. ${ }^{15}$ Targeted interventions can only be developed if the ecology and epidemiology of the disease are well understood. ${ }^{15}$ In the specific case of human and animal trematode infections, which additionally have a zoonotic potential, an ecological understanding of the disease is crucial to identifying the level at which transmission interruption could be initiated. ${ }^{23}$

For fascioliasis in the social-ecological system of mobile pastoralist husbandry systems, control could, for example, be reached through constant transmission reduction over the long term. ${ }^{24}$ Potential leverage points can include decreasing egg contamination of the environment through strategically coordinated temporal treatments of herds. Such efforts should also include behavioural aspects; e.g. adapting water contact patterns and husbandry system could be efficient.

The present study is limited to the report of findings from a mobile pastoralist population encountered in a confined geographical area on the south-eastern Lake Chad area. A larger 
study would be beneficial to confirm whether or not these findings are generalizable to other parts of the Sahel. Additionally, our study suffers from methodological limitations that are generally associated with studies involving mobile populations. Indeed, designing a proper sampling frame is a formidable challenge among highly dynamic mobile pastoralists and their livestock. Repeating stool and urine sampling, which enhances the diagnostic sensitivity of helminth infections, poses difficulties far away from any laboratory facility. ${ }^{25}$ Nevertheless, the simultaneous collection and analyses of human and animal health data can reveal linkages that would not be seen in a separate survey design. ${ }^{26}$ The combined statistical analyses of human and animal data are challenging and depend on the scale of analysis. ${ }^{27}$ The relationship of human and livestock health can often not be related at household, community, and district level, but may show a clear relationship, e.g. for human and animal brucellosis seroprevalence at provincial level. ${ }^{28}$

These findings highlight the potential of One Health studies for a better understanding of human and animal health in socialecological systems. ${ }^{29}$ More specifically, the current research contributes to the evaluation of a One Health study design for parasitic diseases. As elements of a systemic understanding of the transmission dynamics of these water-associated parasitic diseases, our results show cultural- and husbandry system-dependent occurrence of schistosomiasis and fascioliasis. The joint interpretation of the results can provide evidence for potential benefits that can be obtained by joint human and animal intervention strategies for human schistosomiasis and livestock fascioliasis aiming at adding value through close collaboration of the human and livestock health sectors. ${ }^{27,30}$

\section{Conclusions}

Human schistosomiasis, and bovine fascioliasis and schistosomiasis are endemic in the mobile pastoralist populations and their cattle at Lake Chad, specifically in those entering the flood zone of Lake Chad. When stratified by ethnic groups, the prevalence shows similar patterns for human and cattle trematode infections. This illustrates the strong linkage between the traditionally evolved husbandry system and human and animal exposure to trematode infection risks. For both human and animal trematode infections, the most efficacious drugs-praziquantel and triclabendazole-were not available in the study zone. It is conceivable that introducing efficacious treatment against human schistosomiasis and livestock fascioliasis will not only have a positive impact on human and animal health, but also result in economic benefits by improving livestock productivity and reducing treatment costs. Additionally, our study provides an example for the expanded application of a One Health study design beyond zoonotic disease, namely on the research on pathogens sharing distinct characteristics in their disease ecology.

Authors' contribution: $\mathrm{HG}, \mathrm{BNN}, \mathrm{JU}$, and JZ conceived and designed the study protocol; $H G, A A B, B N N$, and DDM implemented the research in Chad; HG and $A A B$ carried out the field work and the parasitological examinations, together with laboratory technicians; $\mathrm{HG}, \mathrm{JH}$, and $\mathrm{JZ}$ analysed and interpreted the data; HG drafted the manuscript; all authors critically revised the manuscript for intellectual content and approved the final manuscript. HG and JZ are the guarantors of the paper.

Acknowledgements: The authors thank the team in Chad for their excellent work in the field laboratory under climatically challenging conditions, and are grateful to all study participants for their enthusiastic collaboration. They acknowledge Rapid Medical Diagnostics (Pretoria, South Africa) for the generous donation of the POC-CCA urine cassette tests.

Funding: This work was funded by the Swiss National Science Foundation (Bern, Switzerland) [grant no. 320030-141246]. The Rudolf Geigy Foundation (Basel, Switzerland) contributed to the training of the Chadian laboratory technicians. The 'Freie Akademische Gesellschaft' (Basel, Switzerland) supported the analysis and writing up of the study.

Competing interests: None declared.

Ethical approval: This study obtained ethical clearance from the ethics committee of the Cantons of Basel (EKBB [reference no. 64/13]). In Chad, research permission, including ethical approval, was obtained from the 'Direction Generale des Activités Sanitaires' in N'Djamena [reference no. 343/MSP/SE/SG/DGAS/2013]. All procedures were in accordance with the ethical standards of the Helsinki Declaration (1964, amended most recently in 2008) of the World Medical Association. To each selected group of mobile pastoralists the purpose, procedures, and potential risks and benefits of the study were explained. Decision for participation was taken as a group and informed consent was signed by the group leader, in respect of the pastoralists' culture. Individual participants were informed about the voluntary participation and that withdrawal was possible at any time without further obligations. Participating individuals assented orally.

\section{References}

1 Montavon A, Jean-Richard V, Bechir M et al. Health of mobile pastoralists in the Sahel-assessment of 15 years of research and development. Trop Med Int Health 2013;18:1044-52.

2 Jean-Richard V, Crump L, Abicho AA et al. Prevalence of Fasciola gigantica infection in slaughtered animals in south-eastern Lake Chad area in relation to husbandry practices and seasonal water levels. BMC Vet Res 2014;10:81.

3 De Haan C, Dubern E, Garancher B et al. Pastoralism development in the Sahel: a road to stability? Washington, DC: World Bank, Global Center on Conflict, Security and Development; 2014.

4 Krätli S, Schareika N. Living off uncertainty: the intelligent animal production of dryland pastoralists. Eur J Dev Res 2010;22:605-22.

5 Jean-Richard V, Crump L, Moto DD et al. The use of mobile phones for demographic surveillance of mobile pastoralists and their animals in Chad: proof of principle. Glob Health Action 2014;7:23209.

6 Krauth SJ, Musard C, Traoré SI et al. Access to, and use of, water by populations living in a schistosomiasis and fascioliasis co-endemic area of northern Côte d'Ivoire. Acta Trop 2015;149:179-85.

7 Utzinger J, Becker SL, van Lieshout L et al. New diagnostic tools in schistosomiasis. Clin Microbiol Infect 2015;21:529-42.

8 Scotch M, Odofin L, Rabinowitz P. Linkages between animal and human health sentinel data. BMC Vet Res 2009;5:15.

9 Jean-Richard V, Crump L, Abicho AA et al. Estimating population and livestock density of mobile pastoralists and sedentary settlements in the south-eastern Lake Chad area. Geospat Health 2015;10:307.

10 Flury C, Ngandolo BN, Müller B et al. Molecular characterization of two common Chadian cattle breeds. AGRI 2009;44:67-76. 
11 Quéval R, Petit JP, Tacher G et al. Le Kouri: race bovine du lac Tchad. I. Introduction générale à son étude zootechnique et biochimique: origines et écologie de la race. Rev Elev Med Vet Pays Trop 1971;24:667-87.

12 Milligan P, Njie A, Bennett S. Comparison of two cluster sampling methods for health surveys in developing countries. Int J Epidemiol 2004;33:469-76.

13 Greter H, Cowan N, Ngandolo BN et al. Treatment of human and livestock helminth infections in a mobile pastoralist setting at Lake Chad: attitudes to health and analysis of active pharmaceutical ingredients of locally available anthelminthic drugs. Acta Trop 2017; 175:91-9.

14 Calavas D, Martin PM. Schistosomiasis in cattle in Corsica, France. Emerg Infect Dis 2014;20:2163-4.

15 Webster JP, Gower CM, Knowles SC et al. One health-an ecological and evolutionary framework for tackling neglected zoonotic diseases. Evol Appl 2016;9:313-33.

16 Christensen NO, Mutani A, Frandsen F. A review of the biology and transmission ecology of African bovine species of the genus Schistosoma. Z Parasitenkd 1983;69:551-70.

17 Moné H, Mouahid G, Morand S. The distribution of Schistosoma bovis Sonsino, 1876 in relation to intermediate host mollusc-parasite relationships. Adv Parasitol 1999;44:99-138.

18 Stothard JR, Lockyer AE, Kabatereine NB et al. Schistosoma bovis in western Uganda. J Helminthol 2004;78:281-4.

19 Webster BL, Rollinson D, Stothard JR et al. Rapid diagnostic multiplex PCR (RD-PCR) to discriminate Schistosoma haematobium and S. bovis. J Helminthol 2010;84:107-14.

20 Webster BL, Diaw OT, Seye MM et al. Introgressive hybridization of Schistosoma haematobium group species in Senegal: species barrier break down between ruminant and human schistosomes. PLoS Negl Trop Dis 2013;7:e2110.
21 Holtfreter MC, Moné H, Müller-Stöver I et al. Schistosoma haematobium infections acquired in Corsica, France, August 2013. Euro Surveill 2014;19:20821.

22 Rollinson D, Knopp S, Levitz S et al. Time to set the agenda for schistosomiasis elimination. Acta Trop 2013;128:423-40.

23 Zinsstag J, Fuhrimann S, Hattendorf J et al. Animal-human transmission models. In: Zinsstag J, Schelling E, Waltner-Toews D et al. (eds). One Health: the theory and practice of integrated health approaches. Wallingford: CABI; 2015: pp. 122-33.

24 Gray DJ, McManus DP, Li Y et al. Schistosomiasis elimination: lessons from the past guide the future. Lancet Infect Dis 2010;10:733-6.

25 Knopp S, Mgeni AF, Khamis IS et al. Diagnosis of soil-transmitted helminths in the era of preventive chemotherapy: effect of multiple stool sampling and use of different diagnostic techniques. PLoS Negl Trop Dis 2008;2:e331.

26 Rabinowitz PM, Kock R, Kachani M et al. Toward proof of concept of a One Health approach to disease prediction and control. Emerg Infect Dis 2013;19(12). doi: 10.3201/eid1912.130265.

27 Schelling E, Hattendorf J. One Health study designs. In: Zinsstag J, Schelling E, Waltner-Toews D et al. (eds). One Health: the theory and practice of integrated health approaches. Wallingford: CABI; 2015: pp. 107-21.

28 Bonfoh B, Kasymbekov J, Dürr S et al. Representative seroprevalences of brucellosis in humans and livestock in Kyrgyzstan. Ecohealth 2012;9:132-8.

29 Zinsstag J, Schelling E, Waltner-Toews D et al. From 'one medicine' to 'One Health' and systemic approaches to health and well-being. Prev Vet Med 2011;101:148-56.

30 Zinsstag J, Béchir M, Schelling E et al. Measuring added value from integrated methods. In: Zinsstag J, Schelling E, Waltner-Toews D et al. (eds). One Health: the theory and practice of integrated health approaches. Wallingford: CABI; 2015: pp. 53-9. 